\title{
Measurements of modal symmetry in subwavelength plasmonic slot waveguides
}

\author{
M. Spasenović, ${ }^{\text {a) }}$ D. van Oosten, E. Verhagen, and L. Kuipers \\ FOM Institute for Atomic and Molecular Physics (AMOLF), Science Park 113, 1098 XG Amsterdam, \\ The Netherlands
}

(Received 14 July 2009; accepted 6 October 2009; published online 17 November 2009)

\begin{abstract}
We excite a guided plasmonic mode in slot waveguides of subwavelength width. With a phase- and polarization-sensitive near-field microscope, we measure the electric field of the mode for a range of slot widths from 40 to $120 \mathrm{~nm}$. The field is experimentally found to be antisymmetric across the slot gap. Numerical calculations confirm this symmetry. Calculations also show a confinement of the field to a lateral size $\sim 10$ times smaller than the free-space wavelength. (C) 2009 American Institute of Physics. [doi:10.1063/1.3254246]
\end{abstract}

Surface plasmon polaritons (SPPs) are electromagnetic waves coupled to charge oscillations at a metal-dielectric interface. ${ }^{1}$ Strong electric fields can be achieved by coupling the SPP modes of two parallel metal surfaces. The structure consisting of two metal surfaces with a dielectric in the middle is commonly referred to as a metal-insulator-metal (MIM) slab waveguide, as SPPs are guided in the dielectric core. ${ }^{2-5}$ In contrast to purely dielectric optical waveguides in which some evanescent field inevitably extends into the surrounding material, ${ }^{6}$ most of the electric field in an MIM slab waveguide is confined to the core even for arbitrarily narrow spacings between the two metal surfaces. Similar confinement exists also in triangular channel guides ${ }^{7}$ and a slot in a metal film in which SPPs on the sides of the slot couple to form a guided mode that is confined to the core in two dimensions. ${ }^{8-10}$ The ability to access the waveguide from the air side and introduce materials or particles into the gap opens up many potential applications. The strong field enhancement between two metal surfaces can be used to sense the presence of molecules ${ }^{11}$ or to produce nonlinear effects. ${ }^{12-15}$ The associated small mode volume could be useful for coupling the emission of individual quantum emitters introduced into the gap to guided modes, which may lead to a nanoscopic source of light on a chip. ${ }^{16}$

When considering any near-field interaction between matter and waveguide modes, the distribution and symmetry of the field must be carefully studied. The symmetry of an incident wave must match the symmetry of the desired waveguide mode for optimal input coupling. In this letter we report near-field measurements of the spatial symmetry of a plasmonic slot waveguide mode. We show that there is only one mode in waveguides of height and width considered in this work. The field of this mode is such that it sets up opposite charges across the gap, i.e., the mode is antisymmetric. ${ }^{17}$ Numerical calculations confirm this symmetry and indicate subwavelength confinement of the mode. Although there have been several far-field input/output studies of plasmonic slot waveguides, ${ }^{10,18-21}$ none of those provide direct information about the symmetry of the mode.

In our work, slots are fabricated by focused ion beam (FEI Helios) milling in a layer of gold, which was evaporated by physical vapor deposition on a BK7 glass substrate.

${ }^{a)}$ Electronic mail: marko@amolf.nl.
The thickness of the gold film is $80 \mathrm{~nm}$. Slot widths $w$ range from 40 to $120 \mathrm{~nm}$ with a fixed length of $17 \mu \mathrm{m}$. Continuous wave laser light from a commercial telecom laser is focused to a spot with a diameter of $\sim 10 \mu \mathrm{m}(1 / e$ in amplitude). The light impinges through the glass substrate perpendicular to the gold layer. The free-space wavelength used in the experiment is $1.55 \mu \mathrm{m}$. At this wavelength, the dielectric constant of gold was experimentally determined by ellipsometry to have a value of $-115.67+11.34 i$, and the dielectric constant of the substrate is 2.25 . The light is linearly polarized orthogonal to the long axis of the slot. A phase- and polarization-sensitive near-field microscope $\mathrm{e}^{22,23}$ is used to image the electric field from the air side of the gold film.

Figure 1 shows the measured amplitude and phase of the electric field component orthogonal to the long axis of the slot. The slot extends from the top left to the bottom right corner of the image, making an angle of $\sim 15^{\circ}$ with the $x$-axis. The amplitude [Fig. 1(a)] above the slot peaks in the middle of the slot, corresponding to the excitation beam. A modulation is superimposed on the amplitude with a measured period of $1.0 \pm 0.1 \mu \mathrm{m}$. The phase image [Fig. 1(b)] shows a similar distribution as the amplitude image in the slot area. The wavefronts parallel to the slot are waves with a wavelength of $\sim 1.5 \mu \mathrm{m}$, propagating to the top and bot-

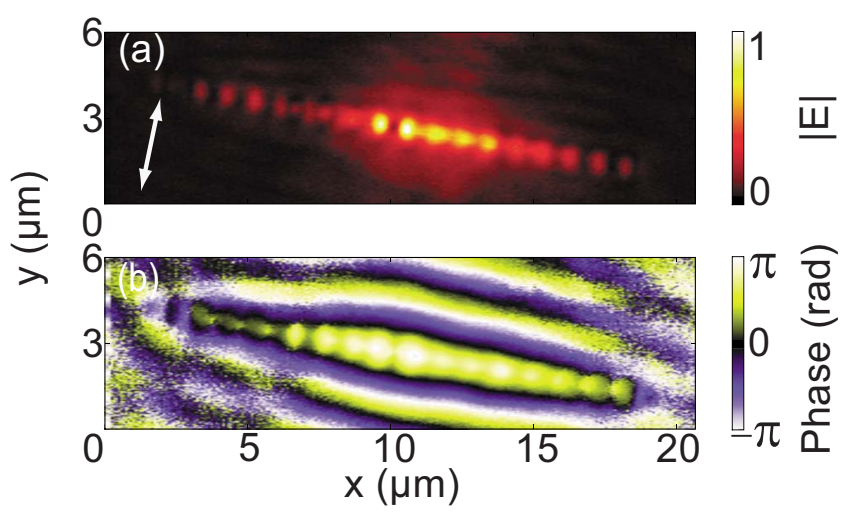

FIG. 1. (Color online) The measured near-field amplitude (a) and phase (b) of the electric field component orthogonal to the long axis of the slot, as indicated by the white arrow. The slot makes an angle of $\sim 15^{\circ}$ with the $x$-axis. The amplitude follows the excitation beam profile with a superimposed modulation. The modulation period is $1.0 \pm 0.1 \mu \mathrm{m}$. 

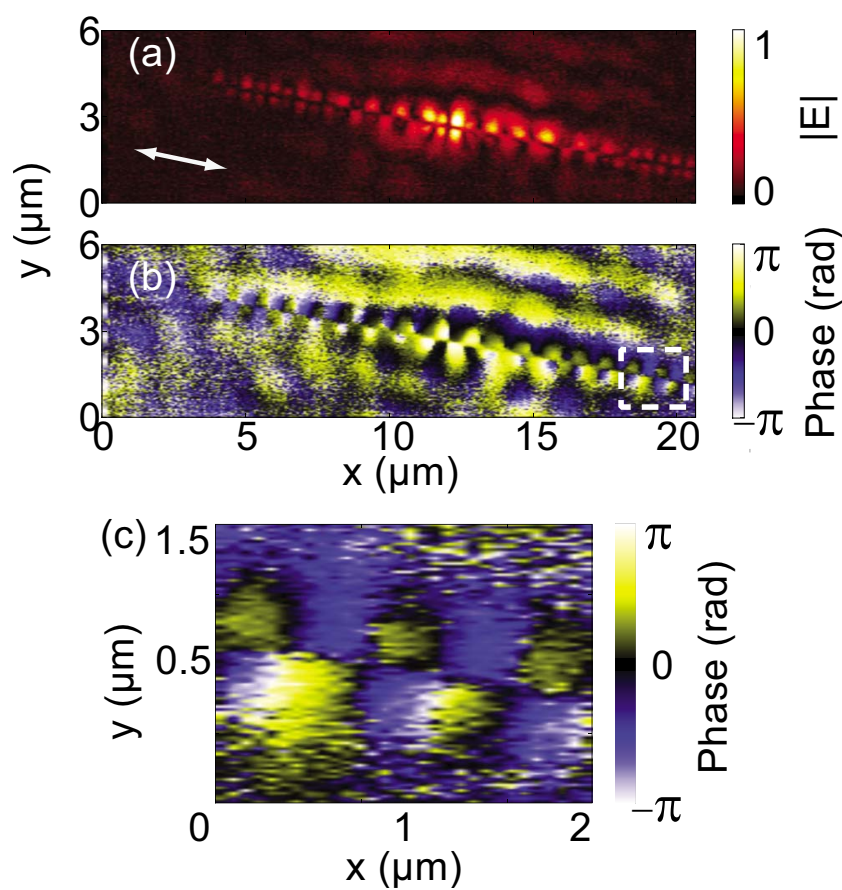

FIG. 2. (Color online) The measured near-field amplitude (a) and phase (b) of the electric field component parallel to the long axis of the slot, as indicated by the white arrow. A close up of the part of the phase image that is surrounded by a dashed white rectangle is shown in (c). The field is antisymmetric, with opposite phases facing each other across the slot. The amplitude is modulated with a period of $0.5 \pm 0.1 \mu \mathrm{m}$.

tom of the image. These are SPPs excited at the slot, propagating on the smooth gold/air interface.

When the component of the electric field parallel to the slot waveguide is measured (Fig. 2), only a modulation is observed. The field exhibits a node along the center of the slot. The modulation period of $0.5 \pm 0.1 \mu \mathrm{m}$, best observed close to the slot ends, is twice as fast as the one observed for the perpendicular field direction [Fig. 1(a)]. The phase in Figs. 2(b) and 2(c) varies with a period of $1.0 \pm 0.1 \mu \mathrm{m}$. The two opposite metal edges of the slot are found to be in antiphase. This indicates that the wave that gives rise to the observed pattern along the slot sets up opposite charges across the slot gap.

To clarify the results further, a finite-element method (FEM) solver (COMSOL) is employed to find the allowed modes of the structure. At widths considered in this work, only one physical solution is found, which has most of the electric field confined to the slot. This is different from the critical slot width of $\sim 1 \mu \mathrm{m}$ found by Pile and co-workers ${ }^{17}$ because the gold film in our case is much thinner than the $400 \mathrm{~nm}$ used in that work. With the calculation, we also verify the measured symmetry. The measured lateral extent of the mode in a $40 \mathrm{~nm}$ wide waveguide $[\sim 600 \mathrm{~nm}$ full width at half maximum (FWHM)] is larger than the calculated one (150 nm FWHM), which we attribute to the finite size of our near-field probe. The electric field is confined to a lateral size $\sim 10$ times smaller than the free-space wavelength.

The match in the calculated and measured modal symmetries indicates that we are indeed observing the plasmonic slot mode in the waveguide. However, the FEM calculation does not provide insight into the excitation mechanism and the origin of the modulation in the amplitude images of Figs. 1(a) and 2(a). To understand the situation further, we perform finite-element time-domain (FDTD) simulations with the Lumerical Solutions FDTD software. In the simulation, we fix the width of the slot to $40 \mathrm{~nm}$ and vary the slot length and the width of the laser spot. It is observed that for our experimental situation, a $17 \mu \mathrm{m}$ long slot and a $10 \mu \mathrm{m}$ wide $(1 / e$ in field) incident beam, the simulation qualitatively reproduces the measured field patterns (Fig. 3). The top part of the figure, which shows the amplitude of the electric field component perpendicular to the slot, shows a weak modulation with a period of $\sim 1.02 \mu \mathrm{m}$, which is approximately $\lambda_{\mathrm{SPP}}$, the calculated wavelength for this slot width. This is the same period that we experimentally observe for the corresponding field component [Fig. 1(a)]. The simulated amplitude of the field component parallel to the slot [Fig. 3(b)] displays two superimposed periods. Besides the periodicity corresponding to $\lambda_{\text {SPP }}$ also observed for the perpendicular field direction in Fig. 3(a), now an additional periodicity corresponding to $\lambda_{\mathrm{SPP}} / 2$ is present, which can be seen most clearly in the middle of the slot. The contrast of the modulation is found to increase for shorter slots and wider laser spots. For longer slots, the contrast of the beating decreases until finally, in an infinitely long slot, the beating vanishes. We therefore conclude that scattering at the slot ends into the plasmonic mode is the excitation mechanism, even though the amplitude of the incident laser at the slot ends is small. The periodicity corresponding to $\lambda_{\mathrm{SPP}} / 2$ in the amplitude pattern is then the result of two counterpropagating modes, each launched by one of the slot ends. Due to the high amplitude of the directly transmitted beam and the relatively weak amplitude of the plasmonic field, the directly transmitted light influences the pattern observed for both field components. The interference between the directly transmitted beam, which has a nearly constant phase along the length of the slot with the plasmonic slot mode, results in the modulation of the amplitude with a periodicity corresponding to

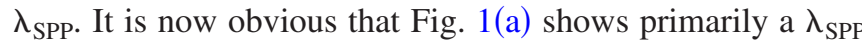
modulation of the amplitude because the transmitted light dominates for the electric field component orthogonal to the long slot axis. On the other hand, for the electric field com-

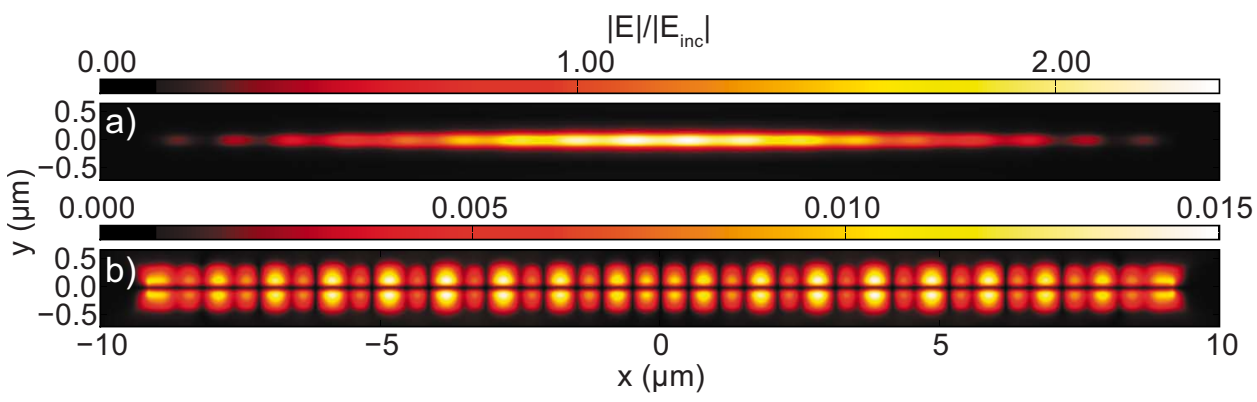

FIG. 3. (Color online) Results of the finite-difference time-domain simulation. Amplitude of the perpendicular (a) and parallel (b) components of the electric field $10 \mathrm{~nm}$ above a slot with a width of $40 \mathrm{~nm}$. The excitation spot has a Gaussian profile with a waist of $10 \mu \mathrm{m}$. The simulated fields show agreement with the measurements in Figs. 1(a) and 2(a). 
ponent parallel to the long slot axis, simulations show that the exact area where the periodicity corresponding to $\lambda_{\mathrm{SPP}}$ dominates the amplitude over the periodicity corresponding to $\lambda_{\mathrm{SPP}} / 2$, as well as the shape of the interference pattern, strongly depends on the position of the center of the beam with respect to the slot ends. This can be understood when we consider that moving the excitation spot closer to or further away from one of the slot ends will change the amplitude and phase relation between the different contributing modes and the transmitted beam. Thus a slight offset in the incident beam with respect to the center of the waveguide can explain the discrepancies between Figs. 2(a) and 3(b).

In conclusion, we have excited a plasmonic mode in a subwavelength slot in a gold film. We observed the electric fields of the mode with phase and polarization sensitivity, showing that the mode is antisymmetric, i.e., opposite charges face each other across the air gap. Due to its high confinement, we expect that this mode can be of great use for two-dimensional plasmonic interconnects, sensing, and quantum plasmonics.

We thank Robert Walters for useful discussions. This work was made possible by the facilities of the Amsterdam nanoCenter. This work is part of the research program of the "Stichting voor Fundamenteel Onderzoek der Materie (FOM)," which is financially supported by the "Nederlandse organisatie voor Wetenschappelijk Onderzoek (NWO)." It is further supported by the Joint Solar Programme (JSP) of FOM, which is cofinanced by Gebied Chemische Wetenschappen of NWO and Stichting Shell Research. Support by the NWO (VICI Grant) is gratefully acknowledged. We acknowledge the support of the EC under the Marie Curie Scheme (Contract No. MEST-CT-2005-021000).
${ }^{1}$ H. Raether, Surface Plasmons (Springer, Berlin, 1988).

${ }^{2}$ S. Collin, F. Pardo, and J.-L. Pelouard, Opt. Express 15, 4310 (2007).

${ }^{3}$ R. Zia, M. D. Selker, P. B. Catrysse, and M. L. Brongersma, J. Opt. Soc. Am. A 21, 2442 (2004).

${ }^{4}$ J. A. Dionne, L. Sweatlock, A. Polman, and H. A. Atwater, Phys. Rev. B 73, 035407 (2006).

${ }^{5}$ F. Villa, T. Lopez-Rios, and L. E. Regalado, Phys. Rev. B 63, 165103 (2001).

${ }^{6}$ A. H. J. Yang, S. D. Moore, B. S. Schmidt, M. Klug, M. Lipson, and D. Erickson, Nature (London) 457, 71 (2009).

${ }^{7}$ S. I. Bozhevolnyi, V. S. Volkov, E. Devaux, J. Y. Laluet, and T. W. Ebbesen, Nature (London) 440, 508 (2006).

${ }^{8}$ G. Veronis and S. Fan, Opt. Lett. 30, 3359 (2005).

${ }^{9}$ L. Liu, Z. Han, and S. He, Opt. Express 13, 6645 (2005).

${ }^{10}$ D. F. P. Pile, T. Ogawa, D. K. Gramotnev, Y. Matsuzaki, K. C. Vernon, K. Yamaguchi, T. Okamoto, M. Haraguchi, and M. Fukui, Appl. Phys. Lett. 87, 261114 (2005).

${ }^{11}$ S. Nie and S. R. Emory, Science 275, 1102 (1997).

${ }^{12}$ A. Bouhelier, M. Beversluis, A. Hartschuh, and L. Novotny, Phys. Rev. Lett. 90, 013903 (2003).

${ }^{13}$ J. A. H. van Nieuwstadt, M. Sandtke, R. M. Harmsen, F. B. Segerink, J. C. Prangsma, S. Enoch, and L. Kuipers, Phys. Rev. Lett. 97, 146102 (2006).

${ }^{14}$ S. Kim, J. Jin, Y. J. Kim, I. Y. Park, Y. Kim, and S. W. Kim, Nature (London) 453, 757 (2008).

${ }^{15}$ A. R. Davoyan, I. V. Shadrivov, and Y. S. Kivshar, Opt. Express 16, 21209 (2008).

${ }^{16}$ Y. C. Jun, R. D. Kekatpure, J. S. White, and M. L. Brongersma, Phys. Rev. B 78, 153111 (2008).

${ }^{17}$ D. F. P. Pile, D. K. Gramotnev, R. F. Oulton, and X. Zhang, Opt. Express 15, 13669 (2007).

${ }^{18}$ L. Chen, J. Shakya, and M. Lipson, Opt. Lett. 31, 2133 (2006).

${ }^{19}$ J. A. Dionne, H. Lezec, and H. Atwater, Nano Lett. 6, 1928 (2006).

${ }^{20}$ H. Miyazaki and Y. Kurokawa, Phys. Rev. Lett. 96, 097401 (2006).

${ }^{21}$ Y. Satuby and M. Orenstein, Appl. Phys. Lett. 90, 251104 (2007).

${ }^{22}$ M. L. M. Balistreri, J. P. Korterik, L. Kuipers, and N. F. van Hulst, Phys. Rev. Lett. 85, 294 (2000).

${ }^{23}$ M. Burresi, R. J. P. Engelen, A. Opheij, D. van Oosten, D. Mori, T. Baba, and L. Kuipers, Phys. Rev. Lett. 102, 033902 (2009). 